\title{
Tale of Constant Adaptation, Revision, and Change of Cancer Therapy during the Ongoing COVID Crisis: Adapting to Cope and Win
}

In the year 1918, there was influenza pandemic, wherein India had the largest number of cases (10-20 million) among all the countries and had the highest case fatality ratio $(4.39 \%)$ worldwide. ${ }^{[1]}$ The total magnitude of estimated deaths globally was 50-100 million. A mathematic model predicted that if similar severity of influenza pandemic would have repeated in 2004, the estimated mortality toll world over would have been 62 million, and with a similar trend as in the past, nearly 14.8 million deaths were estimated in India alone. ${ }^{[2,3]}$

In the wake of a similar pandemic of COVID-19 causing chaos all around the world and claiming thousands of lives, we need to introspect our current position and understand our capacity to "bend the curve" to minimize the magnitude of damage at every cost. ${ }^{[4,5]}$ Fortunately, so far, the situation in India appears better in comparison to many other countries, but the balance is dynamic. Cancer is a disease of the aging and therefore, many of our cancer patients are old. Unfortunately, the severity of COVID-19 illness is also maximum in the elderly and those with comorbid conditions such as uncontrolled hypertension or diabetes and cancer. ${ }^{[1]}$ Hence, to reduce the impact of COVID-19, it is our joint responsibility as oncologists to explicitly communicate our deficiencies, efficiencies, and the ongoing challenges in treatment delivery and come to a consensus as a multidisciplinary team on where we think is most appropriate to draw lines and decide on consistent treatment policies, which are in alignment with the international guidelines, organization's capacity, and safe practices. ${ }^{[2]}$

The great inventions in the medical fields of public health, critical care, and emergency medicine such as vaccines, extracorporeal membrane oxygenation, several antibiotics, antivirals, and targeted therapy have failed to cure critical patients suffering from COVID-19, bolstering the ideology of "Prevention is still better than cure." ${ }^{\text {[3] }}$ Prevention of infection can only be achieved with population-based interventions such as quarantine of the suspected, isolation of the infected, and social distancing to reduce the cross contamination and improving hygiene to eventually "Flatten the otherwise exponentially rising curve of the infected cases." ${ }^{\text {[4,5] }}$ The ultimate goal is to control the pandemic globally and epidemic locally (the strains of the viruses have been found to be different in different countries) by developing of herd immunity but in a controlled manner so as to not overwhelm the health-care sector. ${ }^{[6]}$

Execution of cancer care during the COVID-19 outbreak requires the oncologist to strike a fine balance between selection of treatment that provides meaningful life years and the treatment-related toxicities that make the patient more vulnerable to severe infection of SARS-CoV-2, leading to avoidable mortality. ${ }^{[7]}$ While it may still be comparatively easier for a multidisciplinary cancer care team to come to a consensus on withholding standard treatments, this process becomes challenging through involvement of patients in this decision-making to strike this fine balance. ${ }^{[8]} \mathrm{A}$ pandemic is a protracted dynamic event which can change the existing situations by the day and that can be complicated by a variety of factors other than the virulence such as the region's social, political, and ethical considerations. ${ }^{[9]}$ Hence, the action plans that may be valid today may not hold in future and due cognizance has to be paid to that uncertainty when deciding a road map. ${ }^{[9]}$ Hospitals need to follow the new standards of care developed during the crisis and provide room to adjust them based on the changing volume of patients and severity of infection to successfully triage care..$^{[4,10-13]}$

\section{Cancer and COVID-19}

The association of cancer and COVID-19 is multifaceted and so far, the limited evidence suggests a higher case fatality rate in cancer patients $(6 \%-20 \%)$ as compared to the overall population $(2 \%-3 \%) \cdot{ }^{[1,14,15]}$ There is a plethora of information on SARS-CoV-2 and cancer care guidelines during the COVID-19 outbreak that are updating on a daily or weekly basis. Nevertheless, local and regional factors such as logistics, infrastructure, socioeconomic and sociodemographic structure of the population, the infection rate of the strain of SARS-CoV-2 specific to that region, and the available resources are of paramount importance in adapting guidelines to the different parts of a country. ${ }^{[7,12,13,16-22]}$ Despite this, there are several concerns for the patients as well as physicians in delivering cancer care as follows:

\section{Patient Concerns}

These typically are the fear of the unknown, including that of cancer outcome, probability of infection with coronavirus especially when stepping out for cancer treatment, interaction between cancer and SARS-CoV-2 infection, and feeling more vulnerable due to cancer diagnosis or its treatment. ${ }^{[23,24]}$ The other aspects are anxiety from possible adverse impact of delay in the institution of therapy amidst the crisis and conflicts among patients' and caregivers' choice including the criticality involved with people's end-of-life wishes in a resource-constrained environment. ${ }^{[7,8]}$ Apart from these, a survey by the American Cancer Society (ACS) Cancer Action Network 
showed that more than a quarter of the cancer patients were also worried about job and insurance cover losses, causing difficulty in paying for the cancer treatments. Because most of the cancer patients in India do not have health insurance cover, loss of income would directly impact their out-of-pocket expenditure, further impacting the health-care systems in India beyond the COVID-19 crisis. $^{[25]}$

\section{Concerns for Physicians}

The physician concerns related to infection are the fear of working in a potentially infectious and life-threatening environment with inadequate personal protective equipment (PPE) and potentially increasing infection risk to family. ${ }^{[16]}$ Other concerns are burnout related to long hours of work, shortage of staff as many doctors infected at work get quarantined and a reserve pool needs to be available to take over, rescheduled routines, disturbed sleep from constant psychological pressure to treat patients with mostly noninvasive or supportive care measures, and deal with the paradox of purposely delaying standard therapy even for cancers where overall treatment time is directly correlated with outcomes. ${ }^{[7,16,19]}$ These circumstances can lead to adverse cancer-related outcomes, potentially making physicians vulnerable to litigation and physical violence.

Despite all the concerns, cancer care must continue whenever possible acknowledging the risks associated with it during a COVID-19 outbreak. The following information may be used to select treatments that may be considered safe and appropriate for different cancers in the context of the COVID-19 crisis. $^{[7,13-17,19,26,27]}$

1. Patient factors: The factors that make patients more vulnerable to severe COVID-19 and poor outcomes are extremes of age, uncontrolled chronic medical conditions such as hypertension or diabetes mellitus, poor performance status, lower socioeconomic status, no health insurance, lower education level, and poor support system

2. Tumor factors: These factors may help in prioritizing cancer therapies such as aggressive versus indolent disease, presence of poor prognostic factors, disease potentially threatening to vital organs, or disease progression that may lead to imminent life-threatening event

3. Treatment-related factors: The treatment-related factors that need consideration prior to selecting treatment modalities or initiating therapy are the intent and the expected outcome, the potential effect of delaying standard therapy or using less aggressive therapies, avoiding versus cautious use of intensive chemotherapy protocols which may cause severe myelosuppression, and role of oral metronomic treatment to bridge the gap. It is also equally important to ascertain the availability of infrastructure for limiting cross-contamination in case of asymptomatic infected patients undergoing therapy (as all patients on active cancer treatment will not be tested for infection with SARS-CoV-2 at frequent intervals).

Whenever possible, triaging of patients should be done by a multidisciplinary team and appropriate justification for the same should be documented for future reference and medicolegal purpose. Both patients and physicians should be well versed with the principles of prioritization. ${ }^{[22]}$ Guidelines from the national oncological societies, the oncology peer groups, and the local hospitals based on the international guidelines adapted to the local needs are very helpful. ${ }^{[3,10,12,13,16,19-22,26,28]}$ The NHS-UK takes into the account the probability of survival and potential benefit from therapy and stratifies the patients into six groups. Treatment stratification is by intent and is between curative and palliative, wherein the potential for successful treatment, increasing lifespan, or palliation is graded from $>50 \%, 15 \%$ to $50 \%$, and $15 \% \cdot{ }^{[13]}$ On the other hand, the French guideline also takes into account variables such as age and cancer duration. ${ }^{[21]}$ The ESMO Magnitude of clinical benefit is an objective tool and is worth using to assess the potential benefit of treatment. It is advisable to use online calculators for life expectancy or Cancer and Aging Research Group score for predicting toxicity to provide objectivity in triaging therapy. Once the decision has been made on following through with the treatment of the patient during the COVID-19 outbreak, the following general measures can be practiced to avoid unnecessary contact and cross contamination. ${ }^{[3-5,12-14,20-22,26-32]}$ During the COVID-19 outbreak, the decision to defer or alter standard therapies even though based on international guidelines has to be carefully explained to the patient along with its risks and uncertainties.

\section{Pretreatment Workup and Counseling}

Patients can undergo necessary investigations at a center closer to home and visit the cancer center only for consultation and treatment. Counseling prior to the therapy whether by medical, surgical, or radiation team can be provided using telemedicine units, video/conference calls, or by using prerecorded videos. ${ }^{[25]}$ This ensures minimum contact while still providing all the necessary information effectively. All staff involved in providing active treatment should be provided with appropriate PPE, and utmost care should be taken to disinfect the therapy area before using the facility or devices for the patients and in between patients to avoid inadvertent cross contamination. 45 GSM basic PPE along with mask and gloves for all the housekeeping and administrative staff; 70 GSM PPE with face shield, mask, and gloves for doctors and therapy staff including nurses who come in direct contact of the patient; and the advanced kit of 180 GSM for all intensive care unit (ICU) staff are considered appropriate. However, details of PPE suitable for the setting, activity, and risk of infection suitable for the India have been provided by the 
Directorate General of Health services. ${ }^{[33]}$ Despite all these measures, it is prudent to maintain social distancing in the patient waiting areas, day-care facilities, near the pharmacy and billing departments, and other areas within the hospital premises.

\section{Principles for Choosing Appropriate Systemic Therapy}

Wherever appropriate, de-escalation strategies should be executed such as avoidance of intravenous route for systemic therapies as well as supportive care drugs and use of oral or subcutaneous route such as oral metronomic chemotherapy and oral antibiotics or antifungals and minimization of blood and platelet transfusions. Avoiding or cautious use of intensive chemotherapy protocols with a propensity cause severe myelosuppression or immunosuppression by means of specific T-cell suppression or B-cell immunomodulation (e.g. bendamustine and fludarabine), or substitution with preferably oral metronomic treatment to bridge the gap, may be opted. Use of single-agent checkpoint inhibitor rather than dual therapy as in melanoma and other indications can reduce the toxicity and cost of treatment. ${ }^{[34]}$ At present, preemptive testing for COVID-19 in asymptomatic patients undergoing chemotherapy is not universally recommended. First, there is no evidence that such testing reduces either transmission to other patients or prevents COVID-19 disease in patients undergoing chemotherapy. Second, the availability of tests is limited and should be restricted to those individuals who merit testing as per the national criteria. ${ }^{[35]}$

The therapy interval can be prolonged and less intensive (3- or 4-weekly regimens instead of dose-dense 2-weekly regimens), prophylactic growth factor use may be encouraged, and long-interval prescription refill should be provided to limit hospital visits. ${ }^{[12,36]}$ Because hydroxychloroquine and azithromycin are used for the treatment of severe COVID-19, drugs such as anthracyclines, tyrosine kinase inhibitors, trastuzumab, and anti-vascular endothelial growth factor agents notorious for QTc prolongation should be used with due precaution and monitoring. Palliative intent treatment with good disease control/stable disease can be given treatment holidays. Low-risk febrile neutropenia may be managed on an outpatient basis and in local community clinics. Minimal investigations between treatment cycles and response assessment should be done only when suspecting progression. . $10,16,18,19,34,36,37]^{2}$

\section{Principles to Guide Cancer Surgery}

The calculus of risk-benefit ratio of cancer surgery has completely changed and become far more complicated than it was due to the addition of a new dimension of risk of infection and mortality by COVID-19. Two additional components that need to factored into this already-complicated equation to decide appropriate surgery are the stage of the epidemic that the local geographical area is in as that would require the health-care facility to be available for the treatment of COVID-19 patients and the risk of exposure of health-care worker. ${ }^{[38]}$ The points that need to be considered while deciding for cancer surgery are the need of surgery (stratified as emergency and lifesaving; urgent, if not done alters outcome; and possible to delay in less aggressive early stages) and the type of surgery (stratified based on the time taken to perform, days of hospitalization required in the postoperative setting, overall risk of morbidity and mortality with the current general condition, and comorbidity status). ${ }^{[13,15]}$ We recommend to continue performing simple surface surgeries such as the breast and the thyroid, while elective aerosol-generating procedures that put all health-care workers within the operating room, at risk are not encouraged. High-risk surgeries especially in elderly patients are not recommended. When planned for surgery, it is currently still not advisable to test all asymptomatic patients for COVID-19 as recommended by the Indian Council of Medical Research. All the staff members handling the patients are advised to use universal safety precautions and hand hygiene to avoid contaminating the operation theater, recovery, and the ICU areas. Surgical oncology societies such as the ACS and many others including the National Comprehensive Cancer Network (NCCN) have suggested similar guidelines. ${ }^{[36,38]}$ The NCCN additionally mentions factoring in the time-sensitiveness of the procedure and delaying the surgeries until the outbreak is controlled or till the time when the health-care systems can be bolstered to deal with a sudden increase in COVID-19 patients wherever possible. ${ }^{[31]}$

\section{Principles Guiding Radiation Therapy Decisions}

Several radiation and oncology societies and our personal experience have shed light on the judicious use of radiation during this outbreak, and broadly the following principles should guide our decisions. ${ }^{[7,12,18,28,30-32,36,39]}$ Priority should be given to patients that are at the end of their radiation therapy (RT). All efforts must be focused on making the work environment safe for both the patient and the radiation workers and therefore, it is important to minimize contact by reducing the machine setup and treatment time. This can be achieved by limiting the use of complex setups and treatment plans and also utilizing image guidance only when considered necessary. Patients waiting for definitive RT especially for tumors that are sensitive to overall treatment time should be treated on priority over those waiting for RT. Indolent or hormone-responsive cancers such as hormone-responsive luminal A type of early-stage breast cancer or hormone-responsive prostate cancer, should be recommended hormonal therapy for 6-12 weeks until safe to resume RT services. ${ }^{[40,41]}$ It is recommended to defer RT where it produces no survival advantage except for 
providing palliative care. ${ }^{[28,31]}$ Use of altered fractionation schedules such as hypofractionated UK-FAST and FAST FORWARD regimen for breast cancer or SBRT for prostate cancer can greatly save machine time and is strongly recommended. Similarly, altered fractionation may be used instead of using radiosensitizing concurrent chemotherapy wherever appropriate. ${ }^{[40]}$ These two measures may help reduce the number of fractions for head-and-neck and breast cancers, which account for nearly $50 \%$ of the patients in the radiation department in India. It is recommended to give priority to simple, short-course palliative treatment, especially for distressing symptoms such as bleeding, severe pain, or dyspnea.

Brachytherapy which forms an integral part of treatment of cervical and endometrial cancers should be strongly recommended. However, to minimize hospital visits, one application with two treatments can be used provided it is dosimetrically safe. Pediatric radiation treatments requiring daily anesthesia may be attempted under oral sedation to minimize daily invasive procedure or greater machine time. Patients with preexisting poor pulmonary functions/effort tolerance should not be recommended RT treatments known to cause higher rates of radiation pneumonitis. These recommendations for both the patients and healthcare workers are summarized in Table 1.

While these guidelines were evolving, we had already started working toward producing a safe environment for the cancer patients at Tata Memorial Centre. The initial experience of adapting to the new working environment before and after the nationwide lockdown has been summarized below.

\section{Quest Prior to Lockdown}

All the departments had to change the way things worked, and this affected the postgraduate students, consultants, technologists, as well as ancillary staff. The focus in week 1 of the outbreak in India, before the nationwide lockdown, was to reduce the number of patients that came to the hospital to minimize crowding and prevent opportunities for virus transmission. This was accomplished by restricting patient entry in the hospital more than $30 \mathrm{~min}$ prior to the appointment and providing quick follow-ups including prescription refills and travel concessions for the asymptomatic patients at the entry point of the hospital. All the follow-up patients with appointments for the next 6 weeks were then systematically called for a telephonic follow-up by an oncologist. Only those whom were felt to have symptoms warranting a hospital visit were advised to do so. Thus, only patients on active treatment presented to the hospital. Ordering imaging and other investigations (areas identified to have one of the highest thoroughfares after the outpatient department [OPD]) were limited to be used for absolutely essential indications and emergencies. Rather than giving less time per consult, these restrictions made our OPD consultations longer and conversations more challenging.

\section{Struggles during the Lockdown}

The situation changed with the 40-day nationwide lockdown, which included suspension of all means of public transport, allowing only very few patients within the city to reach the hospital. Unlike other countries where public transport remained active, a major

Table 1: Summary of recommendations for patients and physician

\section{For the patients}

1. Social distancing and hand hygiene to be followed by all and at all times

2. Appropriate PPE to be donned by healthcare workers prior to coming in contact with the patients

3. Patients on active treatment or symptomatic followup patients only should be seen in the OPD

4. Maximize appropriate use of telemedicine and virtual tumor boards

5. Order only absolutely essential blood and other investigations

6. Choose the patients wisely by triaging based on age, comorbidity status, tumor type, and risk of severe infection because of the therapy

7. Minimize therapies that increase the risk of cross contamination or ICU admission such as head and neck surgeries and thoracic surgeries

8. Decrease the number of hospital visits by changing the treatment protocol whenever possible, for example, using 3 weekly chemotherapy rather than weekly/2 weekly therapy, changing radiation dose fractionation from daily to weekly, or by using shorter treatment regimens

9. Avoid treatment that do not provide survival benefit but may increase the risk of severe infection in patients

10. Offer palliative therapy judiciously, balancing the risk and benefits

11. Involve the patients and caregivers in the decision-making process when deferring or altering standard therapies due to the COVID19 outbreak

12. If there is a risk of imminent death or severe disability due to progressive cancer, continue treatment even if high risk

\section{For healthcare workers}

1. Use appropriate PPE as per standard national guidelines

2. Arrange centralized resource/website for adequate communication of the dynamic policy decisions/guidelines with the evolving situation

3. Implement proper screening tools

4. Create clear stay at home and return to work guidelines

5. Ensure wellbeing and undertake measures and activities to reduce stress 
challenge for our center was providing safe transport for the hospital staff so that there was minimal disruption in the active treatment of patients. Fewer technicians and ancillary staff forced us to reduce the overall numbers that were treated every day, automatically leading to delay in therapy for most of the patients during the lockdown. All elective aerosol-generating procedures were minimized, whereas surface surgical procedures otherwise considered safe continued to be performed. Systemic palliative and concurrent chemotherapy with higher risk-benefit ratios were deferred, and adjuvant chemotherapy was postponed in most patients. Neoadjuvant chemotherapy continued albeit with caution on intensity and interval between cycles on a case-to-case basis. Instead of cytotoxic chemotherapies, less toxic regimens such as metronomic schedules were practiced as appropriate. ${ }^{[22]}$ Many patients have been called to report after the lockdown to start chemotherapy. However, if there is further extension of the lockdown or India starts to experience rapid community transmission of SARS-CoV-2, the future of systemic chemotherapy under such circumstances may remain uncertain. Palliative treatment was being offered to all the patients whenever indicated.

\section{Preparedness for Longer Lockdown Duration}

Because India has still not seen rapid community-wide transmission due to early lockdown, one may hope for early return to normalcy. However, if it takes longer, then focus should be on building capacity and re-organization of infrastructure to provide continuity of care for the cancer patients. Quarantine and isolation facility for patients and health-care workers need to be identified. Strict rotational duties should be implemented to retain capacity to treat if some health-care workers need to be quarantined. Participation in forming hospital networks such as the National Cancer Grid may help to form robust referral base for future patients such that patients can continue to receive evidence-based similar care. These networks can aid in collectively forming guidelines and pathways to be followed for de-escalation of therapy and similarly chalk out a plan for exiting these pathways when the outbreak comes under control. The short-term and long-term outcomes of the patients treated during this period should guide in developing future research protocols that can help during such pandemics or similar adverse situation.

Simple maneuvers such as continuing social distancing; omitting unnecessary human contact; and practicing visage/ universal precautions, infection control measures (e.g. hand hygiene and cough etiquette), and nutritional diet, can help to conquer COVID with "COVID." "[11] It is a tale of constant adaptation, auditing, revision, and change of cancer therapy during the unprecedented COVID crisis. We not only are adapting to cope but to win!

\section{Acknowledgments}

All the patients and hospital staff that have cooperated at the Tata Memorial Centre to allow smooth functioning of the hospital amidst the COVID-19 chaos.

\section{Rima Pathak', Garvit Chitkara², Padmaj Kulkarni ${ }^{3}$, Gouri Shankar} Bhattacharyya $^{4}$, Sudeep Gupta ${ }^{5}$, Jyoti Bajpai ${ }^{5}$

${ }^{1}$ Department of Radiation Oncology, Tata Memorial Centre, Tata Memorial Hospital, Homi Bhabha National Institute, Mumbai, Maharashtra, India, ${ }^{2}$ Department of Surgical Oncology, Tata Memorial Centre, Tata Memorial Hospital, Homi Bhabha National Institute, Mumbai, Maharashtra, India, ${ }^{3}$ Department of Medical Oncology, Deenanath Mangeshkar Hospital, Pune, Maharashtra, India, ${ }^{4}$ Department of Medical Oncology, Salt Lake City Medical Centre, Kolkata, West Bengal, India, 5epartment of Medical Oncology, Tata Memorial Centre, Tata Memorial Hospital, Homi Bhabha National Institute, Mumbai,

Maharashtra, India

Address for correspondence: Dr. Jyoti Bajpai,

Department of Medical Oncology, Room 1115, $11^{\text {th }}$ Floor, Homi Bhabha Block, Tata Memorial Centre, Tata Memorial Hospital, Homi Bhabha National Institute, Parel, Mumbai - 400 012, Maharashtra, India. E-mail:dr_jyotibajpai@yahoo.co.in

Submitted: 15 -Apr-2020 Revised: 12-May-2020 Accepted: 19-May-2020 Published: 13-Jun-2020

\section{References}

1. Onder G, Rezza G, Brusaferro S. Case-fatality rate and characteristics of patients dying in relation to COVID-19 in Italy. JAMA 2020. doi: 10.1001/jama.2020.4683. [Online ahead of print].

2. Kant L, Guleria R. Pandemic Flu, 1918: After hundred years, India is as vulnerable. Indian J Med Res 2018;147:221.

3. Management of Patients with Confirmed 2019-nCoV. CDC. Available from: https://www.cdc.gov/coronavirus/2019-ncov/hcp/ clinical-guidance-management-patients.html. [Last accessed on 2020 Apr 09].

4. European Centre for Disease Prevention and Control. Considerations Relating to Social Distancing Measures in Response to COVID-19 - Second Update. Stockholm; 2020. Available from: https://www.ecdc.europa.eu/ sites/default/files/documents/covid-19-social-distan cing-measuresg-guide-second-update.pdf. [Last accessed on 2020 Apr 09].

5. World Health Organization. Managing Epidemics: Key Facts about Major Deadly Diseases. Available from: https://www. who.int/emergencies/diseases/managing-epidemics-interactive. pdf. [Last accessed on 2020 Apr 09].

6. Kwok KO, Lai F, Wei WI, Shan Wong SY, Tang JW. Herd immunity - Estimating the level required to halt the COVID-19 epidemics in affected countries. J Infect 2020;80:e32-3.

7. Hanna TP, Evans GA, Booth CM. Cancer, COVID-19 and the precautionary principle: Prioritizing treatment during a global pandemic. Nat Rev Clin Oncol 2020;17:268-70.

8. Lewis MA. Between Scylla and Charybdis - Oncologic decision making in the time of Covid-19. N Engl J Med 2020. pii: 2006588.

9. Shearer FM, Moss R, McVernon J, Ross JV, McCaw JM. 
Infectious disease pandemic planning and response: Incorporating decision analysis. PLoS Med 2020;17:e1003018.

10. Tsang J, Bajpai J. Novel coronavirus infection - Knowns and unknowns with particular reference to oncology - Combating against COVID-19 with "COVID." Indian J Med Paediatr Oncol 2020;41:108-20.

11. Chandra S, Kassens-Noor E. The evolution of pandemic influenza: Evidence from India, 1918-19. BMC Infect Dis 2014;14:510.

12. Shankar A, Saini D, Roy S, et al.: Cancer Care Delivery Challenges Amidst Coronavirus Disease-19 (COVID-19) Outbreak: Specific Precautions for Cancer Patients and Cancer Care Providers to Prevent Spread. [Internet]. Asian Pac J Cancer Prev 2020;21:569-73.

13. National Health Service United Kingdom: NHS England's Clinical Guide for the Management of Cancer Patients during the Coronavirus Pandemic. Available from: https://www.england.nhs. uk/coronavirus/cancer/. [Last accessed on 2020 Apr 09].

14. Xia Y, Jin R, Zhao J, Li W, Shen H. Risk of COVID-19 for patients with cancer. Lancet Oncol 2020;21:e180.

15. Liang W, Guan W, Chen R, Wang W, Li J, Xu K, et al. Cancer patients in SARS-CoV-2 infection: A nationwide analysis in China. Lancet Oncol 2020;21:335-7.

16. COVID-19 Provider and Practice Information. ASCO. Available from: https://www.asco.org/asco-coronavirus-information/ provider-practice-preparedness-covid-19. [Last accessed on 2020 Apr 09].

17. Zhou F, Yu T, Du R, Fan G, Liu Y, Liu Z, et al:: Clinical course and risk factors for mortality of adult inpatients with COVID-19 in Wuhan, China: A retrospective cohort study. Lancet 2020;395:1054-62.

18. Wu Z, McGoogan JM. Characteristics of and important lessons from the coronavirus disease 2019 (COVID-19) Outbreak in China: Summary of a report of 72314 cases from the Chinese center for disease control and prevention. JAMA - J Am Med Assoc 2020;323:1239-42.

19. Supporting Oncology Professionals. ESMO. Available from: https://www.esmo.org/covid-19-and-cancer/supp orting-oncology-professionals. [Last accessed on 2020 Apr 09].

20. Lambertini M, Toss A, Passaro A, Criscitiello C, Cremolini C, Cardone $\mathrm{C}$, et al. Cancer care during the spread of coronavirus disease 2019 (COVID-19) in Italy: Young oncologists' perspective. ESMO Open 2020;5:e000759.

21. You B, Ravaud A, Canivet A, Ganem G, Giraud P, Guimbaud R, et al. The official French guidelines to protect patients with cancer against SARS-CoV-2 infection. Lancet Oncol 2020;21:619-21.

22. National Institute of Health and Care Excellence. Overview | COVID-19 Rapid Guideline: Delivery of Systemic Anticancer Treatments | Guidance | NICE. NICE; 2020. Available from: https://www.nice.org.uk/guidance/ng161. [Last accessed on 2020 May 26].

23. The American Cancer Society Cancer Action Network: COVID-19 Pandemic Impact on Cancer Patients and Survivors Survey Findings Summary; 2020. Available from: https://www. kff.org/other/state-indicator/total-population/. [Last accessed on 2020 Apr 19].

24. American Society of Clinical Oncology. COVID-19 Patient Care Information. American Society of Clinical Oncology; 2020. Available from: https://www.asco.org/asco-coronavirus-inform ation/care-individuals-cancer-during-covid-19. [Last accessed on 2020 Apr 19].

25. Oxford Economics/ Haver Analytics. Global Analysis of Health Insurance in India. https://www.ey.com/Publication/vwLUAssets/ EY-global-analysis-of-health-insurance-in-india/\$File/ey-globalanalysis-of-health-insurance-in-india.pdf. [Last accessed 2020 Apr 19].

26. Burki TK. Cancer guidelines during the COVID-19 pandemic. Lancet Oncol 2020;21:629-30.

27. Cortiula F, Pettke A, Bartoletti M, Puglisi F, Helleday T. Managing COVID-19 in the oncology clinic and avoiding the distraction effect. Ann Oncol 2020;31:553-5.

28. COVID-19 Rapid Guideline: Delivery of Radiotherapy. NICE; 2020.

29. Q and A on Coronaviruses (COVID-19). Available from: https:// www.who.int/news-room/q-a-detail/q-a-coronaviruses. [Last accessed on 2020 Apr 09].

30. Wei $\mathrm{W}$, Zheng $\mathrm{D}$, Lei $\mathrm{Y}$, Wu S, Verma V, Liu Y, et al. Radiotherapy workflow and protection procedures during the coronavirus disease 2019 (COVID-19) outbreak: Experience of the Hubei cancer hospital in Wuhan, China. Radiother Oncol 2020;148:203-10.

31. Simcock R, Thomas TV, Mercy CE, Filippi AR, Katz MA, Pereira IJ, et al. COVID-19: Global radiation oncology's targeted response for pandemic preparedness. Clin Transl Radiat Oncol 2020;22:55-68.

32. Guckenberger M, Belka C, Bezjak A, Bradley J, Daly ME, DeRuysscher $\mathrm{D}$, et al. Practice recommendations for lung cancer radiotherapy during the COVID-19 pandemic: An ESTRO-ASTRO consensus statement. Radiother Oncol 2020. pii: S0167-8140 (20) 30182-1.

33. Ministry of Health and Family Welfare DG of HS. Novel Coronavirus Disease 2019 (COVID-19): Guidelines on Rational Use of Personal Protective Equipment. Available from: https://www.mohfw.gov.in/pdf/ Guidelinesonrationaluseof PersonalProtectiveEquipment.pdf. [Last accessed 2020 May 26].

34. Mailankody S, Bajpai J. Intelligent adaptation to the changing surroundings amidst the COVID-19 pandemic for sarcomas and melanomas. Cancer Res Stat Treat 2020;3 Suppl S1:110-4.

35. Department of Health Research IC of MR: Strategy for COVID19 Testing in India; 2020. Available from: https:// icmr.nic.in/sites/default/files/upload_documents/Strategey_ for_COVI D19_Test_v4_09042020.pdf. [Last accessed on 2020 Apr 20].

36. National Comprehensive Cancer Network: Coronavirus Disease 2019 (COVID-19) Resources for the Cancer Care Community; 2020. Available from: https://www.nccn.org/covid-19/. [Last accessed on 2020 Apr 10].

37. Kulkarni P, Dhanushkodi M. Novel coronavirus severe acute respiratory syndrome coronavirus 2 (coronavirus disease 2019): We shall overcome! Indian J Med Paediatr Oncol 2020;41:103-6.

38. Slater H. ACS Guidelines for Cancer Surgeries During COVID-19 Epidemic; 2020.

39. Gemici C, Yaprak G. Covid-19 outbreak in a major radiation oncology department; which lessons should be taken? Radiother Oncol 2020. doi:10.1016/j.radonc.2020.03.044. [Epub ahead of print].

40. Shuryak I, Hall EJ, Brenner DJ. Optimized hypofractionation can markedly improve tumor control and decrease late effects 
for head and neck cancer. Int $\mathrm{J}$ Radiat Oncol Biol Phys 2019;104:272-8.

41. Dietz JR, Moran MS, Isakoff SJ, Kurtzman SH, Willey SC, Burstein HJ, et al. Recommendations for prioritization, treatment, and triage of breast cancer patients during the COVID-19 pandemic. the COVID-19 pandemic breast cancer consortium. Breast Cancer Res Treat 2020;181:487.
This is an open access journal, and articles are distributed under the terms of the Creative Commons Attribution-NonCommercial-ShareAlike 4.0 License, which allows others to remix, tweak, and build upon the work non-commercially, as long as appropriate credit is given and the new creations are licensed under the identical terms.

\begin{tabular}{|l|l|}
\hline \multicolumn{2}{|c|}{ Access this article online } \\
\hline Quick Response Code: & Website: \\
& www.ijmpo.org \\
\cline { 2 - 2 } & DOl: \\
\hline
\end{tabular}

How to cite this article: Pathak R, Chitkara G, Kulkarni P, Bhattacharyya GS, Gupta S, Bajpai J. Tale of constant adaptation, revision, and change of cancer therapy during the ongoing COVID crisis: Adapting to cope and win. Indian J Med Paediatr Oncol 2020;41:103-9. 